Abstract P1-S2.07 Table 1 Association between selected risk factors and being "STI positive" (testing positive for Chlamydia, Gonorrhoea, Syphilis and/or Trichomoniasis) among female sex workers attending STI testing sites in Germany, $2010(\mathrm{~N}=1142)$

\begin{tabular}{|c|c|c|c|c|}
\hline & \multirow[b]{2}{*}{$\mathbf{N}_{\text {Total }}$} & \multicolumn{3}{|c|}{$\begin{array}{l}\text { STI positive (Chlamydia, Gonorrhoea, } \\
\text { Syphilis and/or Trichomoniasis), } \mathrm{N}=\mathbf{2 3 3}\end{array}$} \\
\hline & & $\overline{N_{\text {Positive }}}$ & OR $(95 \% \mathrm{Cl})$ & p Value \\
\hline \multicolumn{5}{|l|}{ German language } \\
\hline Fluent (R) & 437 & 59 & 1 & \\
\hline Medium & 301 & 34 & $0.82(0.52$ to 1.30$)$ & 0.376 \\
\hline Very poor / None & 375 & 135 & $3.60(2.55$ to 5.10$)$ & 0.000 \\
\hline \multicolumn{5}{|l|}{ Age } \\
\hline Range $15-77$ years & 1142 & 233 & $0.95(0.93$ to 0.97$)$ & 0.000 \\
\hline \multicolumn{5}{|l|}{ Health insurance } \\
\hline No $(\mathrm{R})$ & 450 & 138 & 1 & \\
\hline Yes & 648 & 87 & $\mathbf{0 . 3 5}(0.26$ to 0.47$)$ & 0.000 \\
\hline \multicolumn{5}{|c|}{ Meet clients on the street } \\
\hline No $(\mathrm{R})$ & 995 & 173 & 1 & \\
\hline Yes & 101 & 43 & $3.52(2.30$ to 5.40$)$ & 0.000 \\
\hline \multicolumn{5}{|c|}{ Meet clients through internet or adds } \\
\hline No $(R)$ & 908 & 195 & 1 & 0.002 \\
\hline Yes & 188 & 21 & $0.46(0.28$ to 0.74$)$ & \\
\hline \multicolumn{5}{|c|}{ Number of clients per week } \\
\hline Range $1-75$ & 270 & 37 & $1.02(1.00$ to 1.04$)$ & 0.028 \\
\hline
\end{tabular}

Explanatory factor included as continuous variable in logistic regression.

(R), Reference group.

\section{P1-S2.08 GEOGRAPHY, MOBILITY, WORK VENUE, AND SEXUALLY TRANSMITTED INFECTIONS AMONG 4100 FEMALE SEX WORKERS IN 20 CITIES IN PERU}

doi:10.1136/sextrans-2011-050108.65

${ }^{1} \mathrm{C}$ Mejia, ${ }^{2} \mathrm{P}$ Campos, ${ }^{2} \mathrm{C}$ Carcamo, ${ }^{2} \mathrm{P}$ Garcia, ${ }^{3} \mathrm{~J}$ Hughes, ${ }^{3} \mathrm{D}$ Martin, ${ }^{4} \mathrm{G}$ Garnett, ${ }^{3} \mathrm{~K}$ Holmes. ${ }^{1}$ Battelle, Seattle, USA; ${ }^{2}$ Universidad Peruana Cayetano, Peru; ${ }^{3}$ University of Washington, Seattle, USA; ${ }^{4}$ mperial College, UK

Objectives To estimate and compare prevalences of five STI Chlamydia trachomatis (CT) Neisseria gonorrhoeae (NG), Trichomonas vaginalis (TV), T pallidum (syphilis), and HIV infection for female sex workers (FSW) according to home geographic region and mobility, and to identify other STI risk factors.

Methods Data on geographic region, internal mobility (defined as having engaged in sex work in $>2$ Peruvian cities in the previous 12 months or was working away from their home city at enrolment), sex work characteristics, sociodemographics and, behaviours, were collected together with vaginal and blood specimens from 4100 FSW in 20 Peruvian cities. Multivariate analysis identified factors associated being infected with any of the five STI.

Results FSW from the jungle region had higher prevalences of CT, NG, TV, syphilis, and HIV than FSW from the coastal or highlands regions. In univariate analysis, having worked in $>2$ more cities or working away from home were significantly negatively associated with having any STI. In multivariate analyses the mobility status variable showed a trend towards protective associations; neither remained significant. Risk of any STI was significantly and independently associated with bar/nightclub or street work, being from the jungle region, and not completing high school.

Conclusion Findings suggest that internal mobility does not increase risk among Peruvian FSW. Preventive interventions should focus on FSW from the jungle region, those working in bars/ nightclubs or on the street and those lacking secondary education to halt the spread of STI among FSW and, presumably, to the general population.

\section{P1-S2.09 WHO IS AT HIGHER RISK OF STIS AND HIV-BROTHEL-BASED OR STREET-BASED FEMALE SEX WORKERS? EVIDENCE FROM TWO ROUNDS OF BIO-BEHAVIOURAL SURVEYS}

doi:10.1136/sextrans-2011-050108.66

${ }^{1}$ A Gautam, ${ }^{1} \mathrm{R}$ Adhikary, ${ }^{1} \mathrm{~S}$ Ramanathan, ${ }^{1} \mathrm{P}$ Goswami, ${ }^{1} \mathrm{~S}$ Khobragade, ${ }^{2} \mathrm{~S} M$ Deshpande, ${ }^{2} \mathrm{~S}$ Khazi, ${ }^{2} \mathrm{M}$ M Mainkar, ${ }^{2} \mathrm{R}$ S Paranjape. ${ }^{1} \mathrm{FHI}$, New Delhi, India, ${ }^{2}$ National AIDS Research Institute, NARI, India

Background Female sex workers (FSWs) are considered a high-risk group for HIV and other STIs. Sex work practices of FSWs in Maharashtra are diverse and vary according to the sex work milieu (eg, street-, brothel- and bar-based). We examined changes in HIV and STI prevalence, associated risk behaviours and socio-economic variables among street-based (SB) and brothel-based (BB) FSWs in Maharashtra.

Methods Two rounds of cross-sectional surveys were conducted in 2005 (R1) and 2009 (R2) in selected districts of Maharashtra. Probability sampling methods, such as conventional and time-location cluster sampling, were used to sample 2257 (1212 BB and 1045 SB) FSWs in R1 and 2228 (1182 BB and 1046 SB) FSWs in R2. Sexual behaviours were assessed, blood and urine samples were collected to measure the prevalence of HIV, Neisseria gonorrhoeae (NG), Chlamydia trachomatis (CT) and syphilis. Changes were analysed using bivariate and multivariate methods.

Results HIV prevalence increased significantly among SB FSWs from $17.2 \%$ in $\mathrm{R} 1$ to $24.3 \%$ in $\mathrm{R} 2$ (OR $1.54, \mathrm{p}=0.04$ ), whereas among $\mathrm{BB}$ FSWs, there was no significant change (28.4 in R1 to 28.9 in R2, OR $1.03, \mathrm{p}=0.88$ ). Prevalence of active syphilis (TPHA and RPR positive at any titre) declined among BB FSWs from $15.4 \%$ (R1) to $11.0 \%$ (R2) (OR 0.67, $\mathrm{p}=0.05$ ), while the decline among SB FSW was not significant (R1-13.6\% to R2-10.6\%; OR 0.75, $\mathrm{p}=0.22$ ). NG prevalence fell from $7.7 \%$ (R1) to $3.9 \%$ (R2) (OR 0.48, $\mathrm{p}=0.006$ ) for $\mathrm{BB}$ FSWs, whereas for SB FSWs, it declined from $7.2 \%$ in R1 to $4.2 \%$ in R2 (OR 0.56, $\mathrm{p}=0.07$ ). No significant change was observed in the prevalence of CT in either group. Self-perceived risk of HIV among BB FSWs increased from $52.5 \%$ (R1) to $62.5 \%$ (R2), (OR 1.5, $\mathrm{p}=0.02)$; whereas among SB FSWs, it was unchanged (49.8\% in R1 to $50.3 \%$ in $\mathrm{R} 2$; OR $1.02, \mathrm{p}=0.23)$. The proportion of illiterate $\mathrm{BB}$ FSWs decreased from $80.1 \%$ (R1) to $74.3 \%$ (R2), (OR $0.72, p=0.02$ ) while for SB FSWs, illiteracy increased significantly from $64.4 \%$ (R1) to $77.1 \%$ (R2), (OR 1.86, $\mathrm{p}<0.001$ ). The proportion of FSWs having more than 10 clients per week was same over both rounds for BB FSWs but for SB FSWs, it increased significantly to $49.9 \%$ (R2) from $32.8 \%$ (R1) (OR 2.04, p<0.001).

Conclusion SB FSWs are at higher risk of contracting HIV and other STIs and are more vulnerable compared to BB FSWs. Prevention interventions must account for differences in profiles, risk behaviours and sex work typology to customise strategies for these groups for maximum impact.

\section{P1-S2.10 FEMALE CLIENTS AND PARTNERS OF MSM SEX WORKERS IN A RESOURCE-POOR SETTING, WEST AFRICA}

doi:10.1136/sextrans-2011-050108.67

${ }^{1} 0$ Busari, ${ }^{2} \mathrm{M}$ Nakayima, ${ }^{3} \mathrm{~A}$ Busari. ${ }^{1}$ Federal Medical Centre, Ido-Ekiti, Nigeria; ${ }^{2}$ The AIDS Support Organization, Masaka, Uganda; ${ }^{3}$ Care AIDS International, Nigeria

MSM are becoming increasingly important in HIV epidemics in Africa and their population is growing in Nigeria. However, there's paucity of data on HIV epidemics in MSM in Nigeria and West Africa region. Though HIV transmission in Africa is mainly heterosexual, interactions between HIV epidemics in MSM and 